\title{
Variational Density Fitting with a Krylov Subspace Method
}

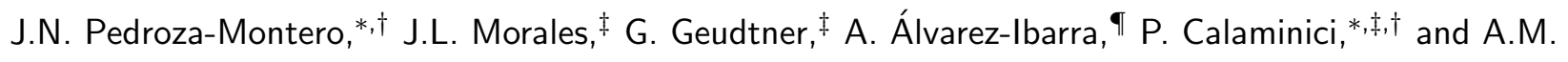
Köster*,+, †

†Programa de Doctorado de Nanociencias y Nanotecnologías, CINVESTAV, Av. Instituto Politécnico Nacional 2508, México

†Departamento de Química, CINVESTAV,

Av. Instituto Politécnico Nacional 2508, México

【Laboratoire de Chimie Physique, Université Paris Sud, CNRS, Université Paris Saclay. 15 avenue Jean Perrin, F91405 Orsay, France

E-mail: npedroza@cinvestav.mx; pcalamin@cinvestav.mx; akoster@cinvestav.mx

\section{Supporting Information}

- Schemes S1, S2 and S3 show the MINRES iterative algorithm alongside its dependencies as implemented in deMon2k.

- Double asymptotic expansion of two-center electron repulsion integrals over atom centered primitive Hermite Gaussian type auxiliary functions.

- Figure S1 depicts a semi-log plot of serial and parallel density fitting timings versus the number of auxiliary functions for a set of n-alkane chains from 20 to 100 carbon atoms.

- Tables S1 and S2 list the SCF cycles for the single point benchmark calculations reported in Table 1 and 2 . 
Scheme S1: Algorithm MINRES for solving $\mathbf{G x}=\mathbf{J}$. It estimates $\phi=\left\|\mathbf{r}^{(k)}\right\|, \psi=$ $\left\|\mathbf{G r} \mathbf{r}^{(k)}\right\|$,

$\chi=\left\|\mathbf{x}^{(k)}\right\|, \mathcal{A}=\|\mathbf{G}\|, \kappa=\operatorname{cond}(\mathbf{G})$.

$\operatorname{MINRES}(\mathbf{G}, \mathbf{J}$, maxit $) \longrightarrow \mathbf{x}, \phi, \psi, \chi,\|\mathbf{G}\|, \kappa$

$\beta_{1}=\|\mathbf{J}\|, \quad \mathbf{v}^{(0)}=\mathbf{0}, \quad \beta_{1} \mathbf{v}^{(1)}=\mathbf{J}, \quad \phi_{0}=\tau_{0}=\beta_{1}, \quad \chi_{0}=0, \quad \kappa=1$

$\delta_{1}^{(1)}=\gamma_{\min }=0, \quad c_{0}=-1, \quad s_{0}=0, \quad \mathbf{d}^{(0)}=\mathbf{d}^{(-1)}=\mathbf{x}^{(0)}=\mathbf{0}, \quad k=1$

while no stopping condition is true

LanczosStep $\left(\mathbf{G}, \mathbf{v}^{(k)}, \mathbf{v}^{(k-1)}, \beta_{k}\right) \rightarrow \alpha_{k}, \beta_{k+1}, \mathbf{v}^{(k+1)}$

$\delta_{k}^{(2)}=c_{k-1} \delta_{k}^{(1)}+s_{k-1} \alpha_{k}, \quad \gamma_{k}^{(1)}=s_{k-1} \delta_{k}^{(1)}-c_{k-1} \alpha_{k}$

$\epsilon_{k+1}^{(1)}=s_{k-1} \beta_{k+1}, \quad \delta_{k+1}^{(1)}=-c_{k-1} \beta_{k+1}$

SymOrtho $\left(\gamma_{k}^{(1)}, \beta_{k+1}\right) \rightarrow c_{k}, s_{k}, \gamma_{k}^{(2)}$

$\tau_{k}=c_{k} \phi_{k-1}, \quad \phi_{k}=s_{k} \phi_{k-1}, \quad \psi_{k-1}=\phi_{k-1} \sqrt{\left[\gamma_{k}^{(1)}\right]^{2}+\left[\delta_{k+1}^{(1)}\right]^{2}}$

if $k=1 \quad \mathcal{A}_{k}=\sqrt{\alpha_{1}^{2}+\beta_{2}^{2}} \quad$ else $\mathcal{A}_{k}=\max \left\{\mathcal{A}_{k-1}, \sqrt{\beta_{k}^{2}+\alpha_{k}^{2}+\beta_{k+1}^{2}}\right\} \quad$ end

if $\gamma_{k}^{(2)} \neq 0$,

$$
\begin{aligned}
& \mathbf{d}^{(k)}=\left(\mathbf{v}^{(k)}-\delta_{k}^{(2)} \mathbf{d}^{(k-1)}-\epsilon_{k}^{(1)} \mathbf{d}^{(k-2)}\right) / \gamma_{k}^{(2)}, \quad \mathbf{x}^{(k)}=\mathbf{x}^{(k-1)}+\tau_{k} \mathbf{d}^{(k)}, \quad \chi_{k}=\left\|\mathbf{x}^{(k)}\right\| \\
& \gamma_{\min }=\min \left\{\gamma_{\min }, \quad \gamma_{k}^{(2)}\right\}, \quad \kappa=\mathcal{A}_{k} / \gamma_{\min }
\end{aligned}
$$

end

$k \leftarrow k+1$

end

$\mathbf{x}=\mathbf{x}^{(k)}, \quad \phi=\phi_{k}, \quad \psi=\phi_{k} \sqrt{\left[\gamma_{k+1}^{(1)}\right]^{2}+\left[\delta_{k+2}^{(1)}\right]^{2}}, \quad \chi=\chi_{k}, \quad \mathcal{A}=\mathcal{A}_{k}$

end

Scheme S2: Algorithm LanczosStep to generate a new Lanczos vector.

\begin{tabular}{|l|} 
LanczosStep $\left(\mathbf{G}, \mathbf{v}^{(k)}, \mathbf{v}^{(k-1)}, \beta_{k}\right) \rightarrow \alpha_{k}, \beta_{k+1}, \mathbf{v}^{(k+1)}$ \\
$\mathbf{p}^{(k)}=\mathbf{G} \mathbf{v}^{(k)}, \quad \alpha_{k}=\left[\mathbf{v}^{(k)}\right]^{T} \mathbf{p}^{(k)}, \quad \mathbf{p}^{(k)} \leftarrow \mathbf{p}^{(k)}-\alpha_{k} \mathbf{v}^{(k)}$ \\
$\mathbf{v}^{(k+1)}=\mathbf{p}^{(k)}-\beta_{k} \mathbf{v}^{(k-1)}, \quad \beta_{k+1}=\left\|\mathbf{v}^{(k+1)}\right\|$ \\
if $\beta_{k+1} \neq 0, \quad \mathbf{v}^{(k+1)} \leftarrow \mathbf{v}^{(k+1)} / \beta_{k+1} \quad$ end \\
\hline
\end{tabular}


Scheme S3: Algorithm SymOrtho performing a Givens rotation.

SymOrtho $(a, b) \rightarrow c, s, r$
if $b=0$
$\quad s=0, r=|a|$, if $\quad a=0, c=1 \quad$ else $c=\operatorname{sign}(a) \quad$ end
elseif $a=0$
$\quad c=0, s=\operatorname{sign}(b), r=|b|$
elseif $|b| \geq|a|$
$\tau=a / b, s=\operatorname{sign}(b) / \sqrt{1+\tau^{2}}, c=s \tau, r=b / s$
elseif $|a|>|b|$
$\quad \tau=b / a, c=\operatorname{sign}(a) / \sqrt{1+\tau^{2}}, s=c \tau, r=a / c$
end


Double asymptotic expansion of two-center ERIs over atom centered primitive Hermite Gaussian type auxiliary functions

For the variational fitting of the Coulomb potential in deMon2k atom centered primitive Hermite Gaussians, denoted by Latin letters with a bar, are used as auxiliary functions. They are grouped together in sets with common exponents. ${ }^{1}$ With these functions an element of the Coulomb matrix is given as:

$$
G_{\bar{c} \bar{d}}=\langle\overline{\mathbf{c}}|| \overline{\mathbf{d}}\rangle=\iint \frac{\overline{\mathbf{c}}(\vec{r}) \overline{\mathbf{d}}\left(\vec{r}^{\prime}\right)}{\left|\vec{r}-\vec{r}^{\prime}\right|} d \vec{r} d \vec{r}^{\prime}
$$

This two-electron, two-center ERI can be written as an one-electron integral by:

$$
\langle\overline{\mathbf{c}} \| \overline{\mathbf{d}}\rangle=\int \overline{\mathbf{c}}(\vec{r}) \phi_{\bar{d}}(\vec{r}) d \vec{r}
$$

The here introduced electrostatic potential, $\phi_{\bar{d}}(\vec{r})$, of the primitive Hermite Gaussian auxiliary function $\bar{d}(\vec{r})$ is defined as:

$$
\phi_{\bar{d}}(\vec{r})=\int \frac{\overline{\mathbf{d}}(\vec{r})}{\left|\vec{r}-\vec{r}^{\prime}\right|} d \vec{r}^{\prime}
$$

Based on the asymptotic expansion of the Boys function, we find as asymptotic expansion for this potential: ${ }^{2,3}$

$$
\phi_{\bar{d}}(\vec{r}) \sim\left(\frac{\pi}{\bar{\zeta}_{\bar{d}}}\right)^{3 / 2}\left(\frac{\partial}{\partial D_{x}}\right)^{\bar{d}_{x}}\left(\frac{\partial}{\partial D_{y}}\right)^{\bar{d}_{y}}\left(\frac{\partial}{\partial D_{z}}\right)^{\bar{d}_{z}} \frac{1}{|\vec{r}-\vec{D}|}
$$

Using the asymptotic expansion and the operator definition

$$
\hat{\mathcal{A}}_{D}(\overline{\mathbf{d}})=\left(\frac{\partial}{\partial D_{x}}\right)^{\bar{d}_{x}}\left(\frac{\partial}{\partial D_{y}}\right)^{\bar{d}_{y}}\left(\frac{\partial}{\partial D_{z}}\right)^{\bar{d}_{z}} \frac{1}{|\vec{r}-\vec{D}|},
$$


we obtain as asymptotic expansion for the Coulomb matrix elements defined in Eq. (1) and $(2)$ :

$$
\langle\overline{\mathbf{c}}|| \overline{\mathbf{d}}\rangle \sim\left\langle\overline{\mathbf{c}} \mid \hat{\mathcal{A}}_{D}(\overline{\mathbf{d}})\right\rangle
$$

Thus, the first asymptotic expansion of the $\langle\overline{\mathbf{c}} \| \overline{\mathbf{d}}\rangle$ ERI yields a nuclear atraction like integral at nucleus D where the asymptotically expanded auxiliary function potential, $\phi_{\bar{d}}(\vec{r})$, is centered. Because $\overline{\mathbf{c}}(\vec{r})$ is a primitive Hermite Gaussian function the nuclear atraction integral in Eq. (5) can be rewritten as:

$$
\begin{aligned}
\left\langle\overline{\mathbf{c}} \mid \hat{\mathcal{A}}_{D}(\overline{\mathbf{d}})\right\rangle & =\widehat{\mathcal{D}}_{C}(\overline{\mathbf{c}}) \widehat{\mathcal{D}}_{D}(\overline{\mathbf{d}})\left\langle\overline{\mathbf{s}} \mid \hat{\mathcal{A}}_{D}(\mathbf{0})\right\rangle, \\
& =\widehat{\mathcal{D}}_{C}(\overline{\mathbf{c}}) \widehat{\mathcal{D}}_{D}(\overline{\mathbf{d}}) \frac{2 \pi}{\zeta_{\bar{c}}} F_{0}\left[\zeta_{\bar{c}}(\vec{C}-\vec{D})^{2}\right]
\end{aligned}
$$

Here $\widehat{\mathcal{D}}_{C}$ and $\widehat{\mathcal{D}}_{D}$ denote the operators

$$
\widehat{\mathcal{D}}_{C}(\overline{\mathbf{c}}) \equiv\left(\frac{\partial}{\partial C_{x}}\right)^{\bar{c}_{x}}\left(\frac{\partial}{\partial C_{y}}\right)^{\bar{c}_{y}}\left(\frac{\partial}{\partial C_{z}}\right)^{\bar{c}_{z}}
$$

and

$$
\widehat{\mathcal{D}}_{C}(\overline{\mathbf{c}}) \equiv\left(\frac{\partial}{\partial D_{x}}\right)^{\bar{d}_{x}}\left(\frac{\partial}{\partial D_{y}}\right)^{\bar{d}_{y}}\left(\frac{\partial}{\partial D_{z}}\right)^{\bar{d}_{z}}
$$

Applying once again the asymptotic expansion of the Boys function in Eq. (6) yields as double asymptotic expansion for the two-electron, two-center ERIs of the Coulomb matrix:

$$
\begin{aligned}
\langle\overline{\mathbf{c}} \| \overline{\mathbf{d}}\rangle & \sim\left(\frac{\pi}{\zeta_{\bar{c}}}\right)^{3 / 2}\left(\frac{\pi}{\zeta_{\bar{d}}}\right)^{3 / 2} \widehat{\mathcal{D}}_{C}(\overline{\mathbf{c}}) \widehat{\mathcal{D}}_{D}(\overline{\mathbf{d}}) \frac{1}{|\vec{C}-\vec{D}|} \\
& =(-1)^{\bar{d}}\left(\frac{\pi}{\zeta_{\bar{c}}}\right)^{3 / 2}\left(\frac{\pi}{\zeta_{\bar{d}}}\right)^{3 / 2} \widehat{\mathcal{D}}_{C}(\overline{\mathbf{c}}+\overline{\mathbf{d}}) \frac{1}{|\vec{C}-\vec{D}|} \\
& =(-1)^{\bar{c}}\left(\frac{\pi}{\zeta_{\bar{c}}}\right)^{3 / 2}\left(\frac{\pi}{\zeta_{\bar{d}}}\right)^{3 / 2} \widehat{\mathcal{D}}_{D}(\overline{\mathbf{c}}+\overline{\mathbf{d}}) \frac{1}{|\vec{C}-\vec{D}|}
\end{aligned}
$$

Within the double asymptotic expansion of $\langle\overline{\mathbf{c}} \| \overline{\mathbf{d}}\rangle$ the auxiliary function shells of atoms can be collected together. As a result, the corresponding matrix vector multiplication scales with 
the number of atoms rather than auxiliary functions.

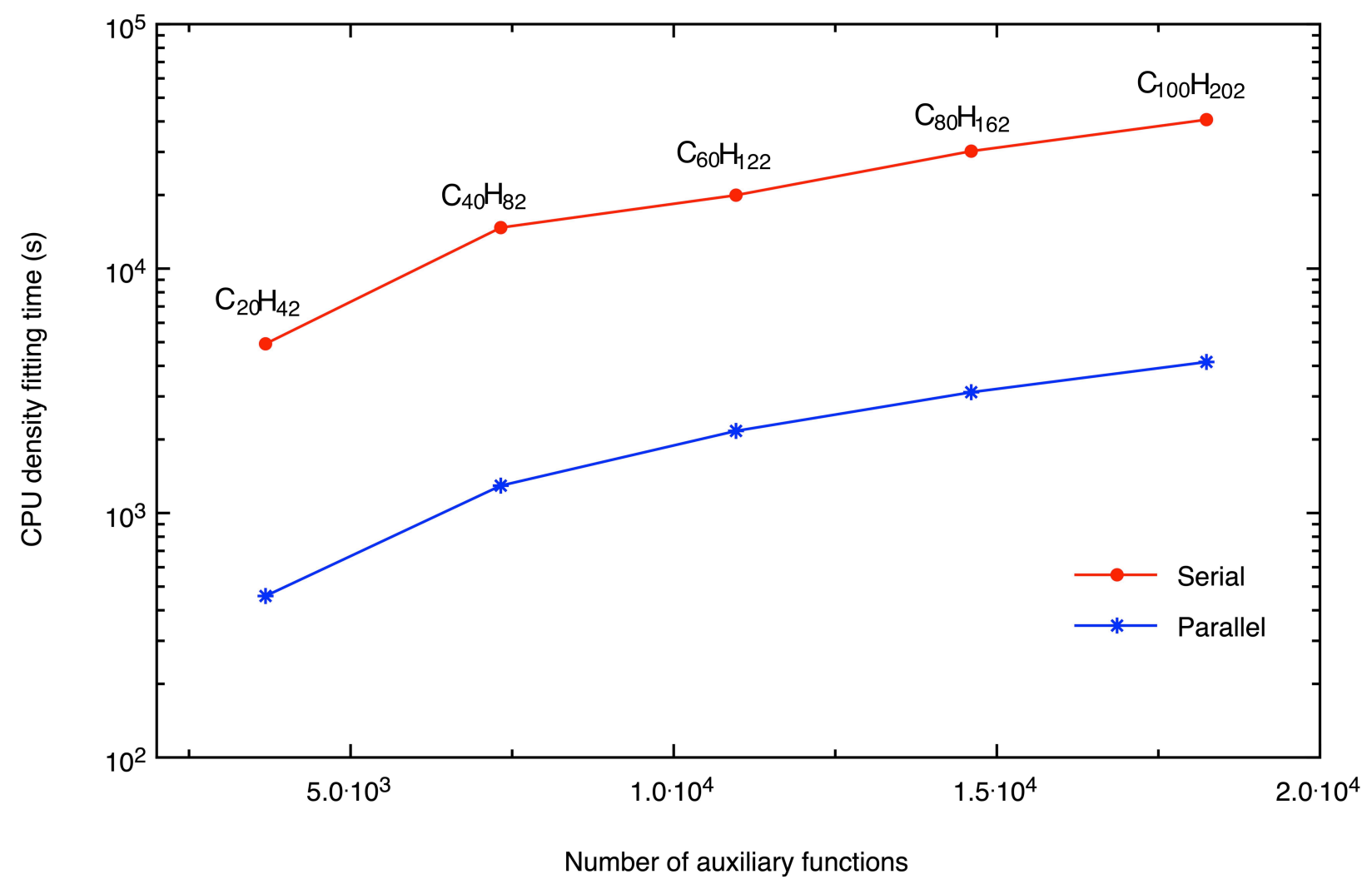

Figure S1: Comparison of serial (red curve) and parallel (blue curve) density fitting timings for small n-alkane chains employing the PBE/aug-cc-pVTZ/GEN-A2* level of theory. The parallel calculations were performed with 12 cores. 
Number of SCF cycles for the single point energy calculations

Table S1: Number of TED and MINRES SCF cycles for single point energy calculations of fullerenes.

\begin{tabular}{|l|c|c|c|c|}
\hline & TED & DIRECT & ASYMPTOTIC & MIXED \\
\hline $\mathrm{C}_{20}$ & 13 & 13 & 13 & 13 \\
\hline $\mathrm{C}_{60}$ & 11 & 11 & 13 & 11 \\
\hline $\mathrm{C}_{180}$ & 14 & 14 & 14 & 14 \\
\hline $\mathrm{C}_{240}$ & 14 & 14 & 14 & 14 \\
\hline $\mathrm{C}_{540}$ & 13 & 15 & 15 & 15 \\
\hline $\mathrm{C}_{720}$ & 15 & 16 & 16 & 15 \\
\hline $\mathrm{C}_{960}$ & 16 & 15 & 15 & 15 \\
\hline
\end{tabular}

Table S2: Number of TED and MINRES SCF cycles for single point energy calculations of water clusters.

\begin{tabular}{|l|c|c|c|c|}
\hline & TED & DIRECT & ASYMPTOTIC & MIXED \\
\hline$\left(\mathrm{H}_{2} \mathrm{O}\right)_{50}$ & 13 & 13 & 13 & 13 \\
\hline$\left(\mathrm{H}_{2} \mathrm{O}\right)_{100}$ & 14 & 15 & 20 & 15 \\
\hline$\left(\mathrm{H}_{2} \mathrm{O}\right)_{200}$ & 15 & 35 & 36 & 35 \\
\hline$\left(\mathrm{H}_{2} \mathrm{O}\right)_{300}$ & 15 & 38 & 38 & 36 \\
\hline$\left(\mathrm{H}_{2} \mathrm{O}\right)_{400}$ & 15 & 39 & 41 & 41 \\
\hline$\left(\mathrm{H}_{2} \mathrm{O}\right)_{500}$ & 16 & 43 & 40 & 39 \\
\hline
\end{tabular}




\section{References}

(1) Calaminici, P.; Janetzko, F.; Köster, A.M.; Mejía-Olvera, R.; Zúñiga-Gutiérrez, B. Density functional theory optimized basis sets for gradient corrected functionals: $3 \mathrm{~d}$ transition metal systems. J. Chem. Phys. 2007, 126, 044108.

(2) Köster, A.M. Hermite Gaussian auxiliary functions for the variational fitting of the Coulomb potential in density functional methods. J. Chem. Phys. 2003 118, 99439951.

(3) Álvarez-Ibarra, A.; Köster, A.M. Double asymptotic expansion of three center electronic repulsion integrals. J. Chem. Phys. 2013, 139, 024102. 